\title{
Rare four leptonic B-mesons decays with a neutrino in final state
}

\author{
Anna Danilina ${ }^{1,2,3, *}$, Nikolay Nikitin ${ }^{1,2,3, * *}$, and Konstantin Toms ${ }^{4}$ \\ ${ }^{1}$ D. V. Skobeltsyn Institute of Nuclear Physics, M. V. Lomonosov Moscow State University, 119991, \\ Moscow, Russia \\ ${ }^{2}$ M. V. Lomonosov Moscow State University, Faculty of Physics, 119991, Moscow, Russia \\ ${ }^{3}$ A. I. Alikhanov Institute for Theoretical and Experimental Physics, 117218 Moscow, Russia \\ ${ }^{4}$ University of New Mexico, Albuquerque, NM 87131
}

\begin{abstract}
.
We revisit prediction for the four - leptonic decay of charged B - mesons in the Standard Model and provide an updated results for branching ratios and various differential distributions. We have taken into account relative phase between intermediate $\rho^{0}(770)$ - and $\omega(782)$ - resonances contributing to the amplitude in the framework Vector Meson Dominance model approach.
\end{abstract}

\section{Introduction}

The study of B-decays provides an excellent opportunity to test and improve our understanding of the Standard Model of Particle Physics and of its limitations. In particular, the LHCb and CMS collaborations are providing us with an impressive amount of experimental information about leptonic B-decays. In the framework of the Standard Model, leptonic decays of the $\mathrm{B}$ - mesons are rare due to the CKM suppression. It makes four - leptonic decay of B meson investigations quite perspective from the point of view of the New Physics searches. These searches are currently being conducted at the LHC. One of them is performed by the LHCb collaboration, where an upper limit on the branching ratio of the $B^{-} \rightarrow \mu^{+} \mu^{-} \bar{v}_{\mu} \mu^{-}$ decay is obtained [1]. We must point out that there is some difference between the experimental result and only theoretical prediction from Ref.[2]. Here we present a more detailed evaluation for the branching ratio of the $B^{-} \rightarrow \mu^{+} \mu^{-} \bar{v}_{\mu} \mu^{-}$decay, that mitigates this difference. In addition, we study series of differential distribution for the $B^{-} \rightarrow \ell^{+} \ell^{-} \bar{v}_{\ell^{\prime}} \ell^{\prime-}$ decay that demonstrate the features of the rare four - leptonic B - decays in the framework of the Standard Model.

\section{Theoretical review of $B^{-} \rightarrow \ell^{+} \ell^{-} \bar{v}_{\ell^{\prime}} \ell^{\prime-}$}

\subsection{The effective Hamiltonian}

The Hamiltonian of the rare four-leptonic decays $B^{-} \rightarrow \ell^{+} \ell^{-} \bar{v}_{\ell^{\prime}} \ell^{\prime-}$ contains a weak and an electromagnetic components and can be expressed as

$$
\mathcal{H}_{\text {eff }}(x)=\mathcal{H}_{\text {weak }}(x)+\mathcal{H}_{\text {em }}(x) .
$$

\footnotetext{
*e-mail: anna.danilina@cern.ch

**e-mail: Nikolai.Nikitine@cern.ch
} 
The contribution from the $b \rightarrow u W^{-} \rightarrow u \ell^{-} \bar{v}_{\ell}$ transitions is

$$
\mathcal{H}_{\text {weak }}(x)=-\frac{G_{F}}{\sqrt{2}} V_{u b}\left(\bar{u}(x) \gamma^{\mu}\left(1-\gamma^{5}\right) b(x)\right)\left(\bar{\ell}(x) \gamma_{\mu}\left(1-\gamma^{5}\right) v_{\ell}(x)\right)+\text { h.c. }
$$

where $u(x)$ and $b(x)$ are quark fields, $\ell(x)$ and $v_{\ell}(x)$ are lepton fields, $G_{F}$ is the Fermi constant, $V_{u b}$ is the corresponding matrix element of the Cabibbo-Kobayashi-Maskawa (CKM) matrix, the matrix $\gamma^{5}$ is defined as $\gamma^{5}=i \gamma^{0} \gamma^{1} \gamma^{2} \gamma^{3}$.

The Hamiltonian of electromagnetic interaction has the form:

$$
\mathcal{H}_{e m}(x)=-e \sum_{f} Q_{f}\left(\bar{f}(x) \gamma^{\mu} f(x)\right) A_{\mu}(x)=-j_{e m}^{\mu} A_{\mu}(x)
$$

where the unitary charge $e=|e|$ is normalized by $e^{2}=4 \pi \alpha_{e m} ; \alpha_{e m} \approx 1 / 137-$ the fine structure constant, $Q_{f}$ - charge of the fermion of the flavor $f$ in units of the unitary charge, $f(x)$ - fermionic field of flavor $f$ and $A_{\mu}(x)$ - four-potential of electromagnetic field.

\subsection{The virtual photon emission from $u$-quark of the B meson}

In accordance with the Hamiltonian structure, we have three types of diagrams, contributing to the amplitude of the $B^{-} \rightarrow \ell^{+} \ell^{-} \bar{v}_{\ell^{\prime}} \ell^{\prime-}$ decays. First of them corresponds to the virtual photon emission from $u$ - quark of the $\mathrm{B}-$ meson. In this case we apply the Vector meson dominance model (VMD), representing the virtual photon contribution as a intermediate $\rho^{0}$ - and $\omega-$ resonances.[Fig. 1]

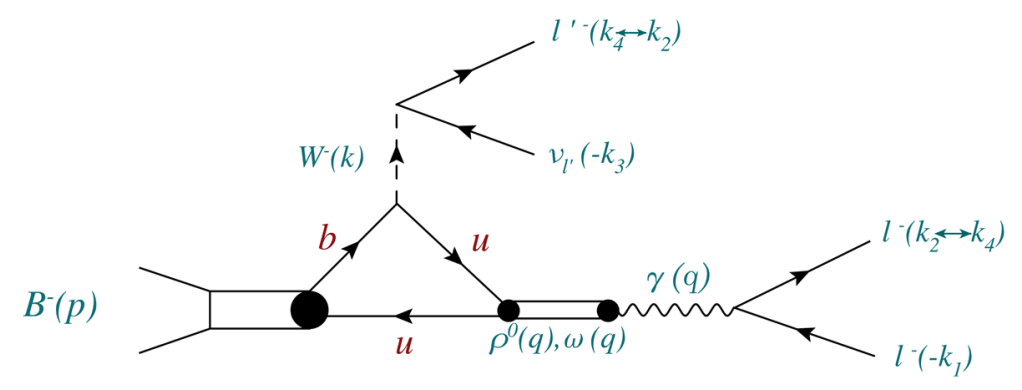

Figure 1. Virtual-photon emitted from the light quark of the B - meson

The hadronic matrix element for the virtual-photon emission from the light quark can be written in terms of three form factors $V^{(i)}\left(k^{2}\right), A_{1}^{(i)}\left(k^{2}\right)$ and $A_{2}^{(i)}\left(k^{2}\right)$ :

$$
M_{f i}^{(u)}=\frac{G_{F}}{\sqrt{2}} 4 \pi \alpha_{e m} V_{u b} \frac{1}{q^{2}} \sum_{i=\rho^{0}, \omega} \frac{f_{V}^{i} M_{2 i} I i}{q^{2}-M_{2 i}^{2}+i \Gamma_{2 i} M_{2 i}} F_{\mu \nu}^{(i)}\left(k^{2}\right)\left(\bar{\ell}\left(k_{2}\right) \gamma^{\nu} \ell\left(-k_{1}\right)\right)\left(\bar{\ell}\left(k_{4}\right) \gamma^{\mu}\left(1-\gamma^{5}\right) \nu\left(-k_{3}\right)\right)
$$

where

$$
F_{\mu \nu}^{(i)}\left(k^{2}\right)=\frac{2 V^{(i)}\left(k^{2}\right)}{M_{1}+M_{2 i}} \epsilon_{\mu \nu k q}-i\left(M_{1}+M_{2 i}\right) A_{1}^{(i)}\left(k^{2}\right) g_{\mu \nu}+2 i \frac{A_{2}^{(i)}\left(k^{2}\right)}{M_{1}+M_{2 i}} q_{\mu} k_{\nu} .
$$

We take into account only the light $\rho^{0}$ - and $\omega$ - mesons contributions, containing $u \bar{u}-$ pair. That was done via isotopic coefficients $I_{i}$ consideration. 


\subsection{The virtual photon emission from $b$-quark of the $\mathbf{B}$ meson}

Virtual photon emission from the heavy quark of B - meson is illustrated in Fig.[ 2]. Here we have an intermediate vector meson $B^{*-}$, decaying to a heavy pseudoscalar meson $B^{-}$ and a virtual photon. This contribution alone is not so significant, but it is essential due to interference between diagrams. The pole at the mass of the $B^{*-}$ meson was included in the pole parametrisation of the form factors of the transitions $B \rightarrow \rho$ and $B \rightarrow \omega$ in the Ref. [3]

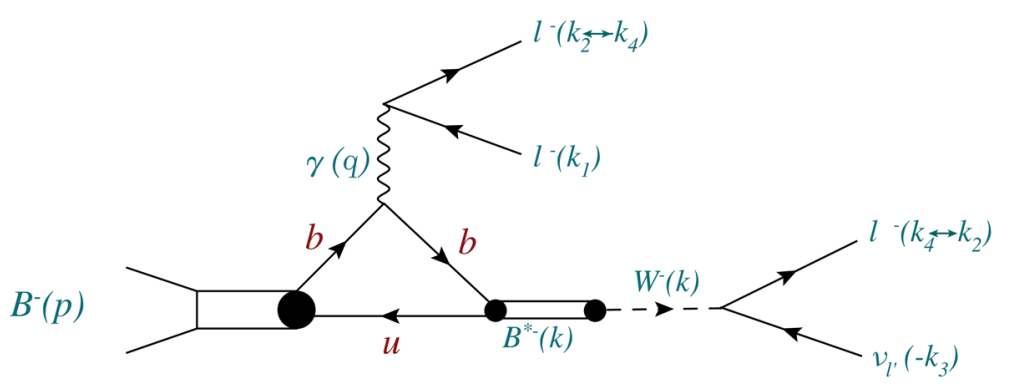

Figure 2. Virtual-photon emitted from the heavy quark of the B - meson

The contribution to matrix element from the diagram in Fig.(2) is:

$$
M_{f i}^{(b)}=\frac{2}{3} \frac{G_{F}}{\sqrt{2}} 4 \pi \alpha_{e m} V_{u b} \frac{1}{q^{2}} \frac{M_{B^{*}} f_{B^{*}}}{k^{2}-M_{B^{*}}^{2}} \frac{V_{b}\left(q^{2}\right)}{M_{1}+M_{B^{*}}} \epsilon_{\mu v p q}\left(\bar{\ell}\left(k_{2}\right) \gamma^{\nu} \ell\left(-k_{1}\right)\right)\left(\bar{\ell}\left(k_{4}\right) \gamma^{\mu}\left(1-\gamma^{5}\right) v\left(-k_{3}\right)\right) .
$$

\subsection{The virtual photon emission from the lepton in final state}

Last diagram, contributing to the amplitude describes the photon emitted from the lepton in the final state. Here we have a photon pole on the $q^{2}$ - variable and lepton masses in final state that could not be reduced, as it is in the other diagrams. The appropriate amplitude can be written in the form

$$
M_{f i}^{(b r m)}=\frac{G_{F}}{\sqrt{2}} 4 \pi \alpha_{e m} V_{u b} \frac{f_{B_{u}}}{q^{2}} g_{\mu \nu}\left(\bar{\ell}\left(k_{2}\right) \gamma^{v} \ell\left(-k_{1}\right)\right)\left(\bar{\ell}\left(k_{4}\right) \gamma^{\mu}\left(1-\gamma^{5}\right) v\left(-k_{3}\right)\right) .
$$

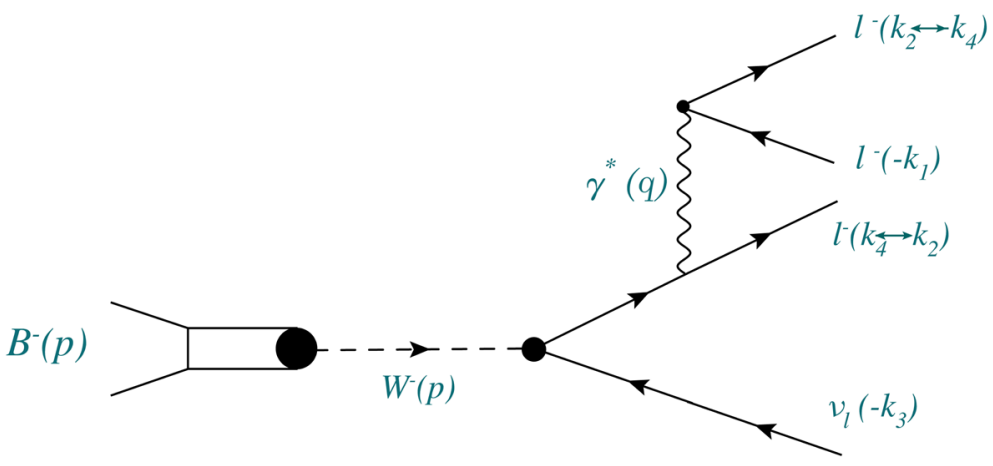

Figure 3. Virtual-photon emitted from the lepton in final state 
Now the full matrix element for the $B^{-} \rightarrow \ell^{+} \ell^{-} \bar{v}_{\ell^{\prime}} \ell^{\prime-}$ decay is

$$
\mathcal{M}_{f i}^{(1234)}=\mathcal{M}_{f i}^{(u)}+\mathcal{M}_{f i}^{(b)}+\mathcal{M}_{f i}^{(b r e m)} .
$$

When there are two identical leptons in the decay, we have the same set of processes, contributing to the amplitude. But in this case number of diagrams is doubled by fermion antisymmetric wave function of identical particles in the final state.

\section{Differential distributions and branching ratios for the $B^{-} \rightarrow \ell^{+} \ell^{-} \bar{v}_{\ell^{\prime}} \ell^{\prime-}$ decays}

\subsection{Branching ratios}

We define the partial width expression of the $B^{-} \rightarrow \ell^{+} \ell^{-} \bar{v}_{\ell^{\prime}} \ell^{\prime-}$ process based on the Eq. 7 as

$$
d \operatorname{Br}\left(B^{-} \rightarrow \ell^{+} \ell^{-} \bar{v}_{\ell^{\prime}} \ell^{\prime-}\right)=\tau_{B^{-}} \frac{\sum_{s_{1}, s_{2}, s_{3}, s_{4}}\left|\mathcal{M}_{f i}^{(1234)}\right|^{2}}{2 M_{1}} d \Phi_{4}^{(1234)}
$$

where $\tau_{B^{-}}$is the lifetime of the $B^{-}$-meson, $\Phi_{4}^{(1234)}$ is the four-particle phase space, and $s_{i}$ is the spin of the fermion in the final state.

And our main result for the branching ratio decay with non - identical particles in final state, for instance for the $B^{-} \rightarrow \mu^{+} \mu^{-} \bar{v}_{e} e^{-}$, is

$$
\operatorname{Br}\left(B^{-} \rightarrow \mu^{+} \mu^{-} e^{-} \bar{v}_{e}\right) \approx 0.6 * 10^{-7} .
$$

When we have identical leptons the in the final state $\left(\ell \equiv \ell^{\prime}\right)$, we obtain an additional interference term, resulting from the double set of diagrams, contributing to the decay amplitude. We thus obtain the differential branching ratio of the decay $B^{-} \rightarrow \mu^{+} \mu^{-} \mu^{-} \overline{v_{\mu}}$ expressed through the branching ratio given by Eq.(8) and the matrix element from Eq.(7) :

$$
\operatorname{Br}\left(B^{-} \rightarrow \ell^{+} \bar{v}_{\ell} \ell^{-} \ell^{-}\right)=\operatorname{Br}\left(B^{-} \rightarrow \ell^{+} \ell^{-} \bar{v}_{\ell^{\prime}} \ell^{\prime-}\right)-\operatorname{Br}_{\text {interf }}\left(B^{-} \rightarrow \ell^{+} \bar{v}_{\ell} \ell^{-} \ell^{-}\right)
$$

where

$$
\begin{aligned}
\operatorname{Br}_{\text {interf }}\left(B^{-}\right. & \left.\rightarrow \ell^{+} \bar{v}_{\ell} \ell^{-} \ell^{-}\right)= \\
& =\frac{\tau_{B^{-}}}{4 M_{1}} \int \sum_{s_{1}, s_{2}, s_{3}, s_{4}}\left(\mathcal{M}_{f i}^{(1234) \dagger} \mathcal{M}_{f i}^{(1432)}+\mathcal{M}_{f i}^{(1432) \dagger} \mathcal{M}_{f i}^{(1234)}\right) d \Phi_{4}^{(1234)}
\end{aligned}
$$

Numerically we have for the interference term :

$$
B r^{(\text {interf })}\left(B^{-} \rightarrow \mu^{+} \mu^{-} \mu^{-} \bar{v}_{\mu}\right) \approx-0.1 * 10^{-7}
$$

And finally for the $B^{-} \rightarrow \mu^{+} \mu^{-} \mu^{-} \overline{v_{\mu}}$ branching ratio:

$$
\operatorname{Br}\left(B^{-} \rightarrow \mu^{+} \mu^{-} \mu^{-} \bar{v}_{\mu}\right) \approx 0.7 * 10^{-7} .
$$

Here it should be noted that the Vector Meson Dominance model used in the matrix element calculation, may produce some uncertainty to the final result. In the VMD model the non - perturbative relative phase between the resonance contributions is equal to zero. In our case there is a phase - shift between intermediate $\rho^{0}$ and $\omega$ mesons, the branching ratio of the 
decay is changed. We perform the calculations for the various phase - shift values to identify the relative phase influence. We define the phase dependence in the resonance amplitude as

$$
M_{f i}^{(\omega)}+e^{i \phi} M_{f i}^{\left(\rho^{0}\right)}
$$

where $\phi$ is the non - perturbative relative phase.

As an illustration, we have considered the $B^{-} \rightarrow \mu^{+} \mu^{-} \bar{v}_{e} e^{-}$decay, but it is also true for the all $B^{-} \rightarrow \ell^{+} \ell^{-} \bar{v}_{\ell^{\prime}} \ell^{\prime-}$ type decay.

Table 1. Branching ratio for the $B^{-} \rightarrow \mu^{+} \mu^{-} \bar{v}_{e} e^{-}$decay at different relative phase

\begin{tabular}{lll}
\hline Amplitude & Phase & Branching ratio \\
\hline$M_{f i}^{(\omega)}+M_{f i}^{\left(\rho^{0}\right)}$ & 0 & $0.6 \times 10^{-7}$ \\
$M_{f i}^{(\omega)}-M_{f i}^{\left(\rho^{0}\right)}$ & $\pi$ & $0.2 \times 10^{-7}$ \\
$M_{f i}^{(\omega)}+i M_{f i}^{\left(\rho^{0}\right)}$ & $\frac{\pi}{2}$ & $0.5 \times 10^{-7}$ \\
$M_{f i}^{(\omega)}-i M_{f i}^{\left(\rho^{0}\right)}$ & $\frac{3 \pi}{2}$ & $0.8 \times 10^{-7}$ \\
\hline
\end{tabular}

From Tab.(1) we can obtain, that the branching ratio $B^{-} \rightarrow \ell^{+} \ell^{-} \bar{v}_{\ell^{\prime}} \ell^{\prime-}$ decay is sensitive to the VMD relative phase between resonances, but even with these corrections the difference between the prediction and experimental upper limit obtained in Ref. [1] is not completely eliminated.

\subsection{Differential distributions}

We plot a set of differential distributions for the decays $B^{-} \rightarrow \mu^{+} \mu^{-} \bar{v}_{e} e^{-}$and $B^{-} \rightarrow \mu^{+} \bar{v}_{\mu} \mu^{-} \mu^{-}$. One-dimensional projections of the double differential distribution $\frac{d^{2} \Gamma}{d x_{12} d x_{34}}$ by $x_{12}$ and $x_{34}$ are given in Fig. 4.

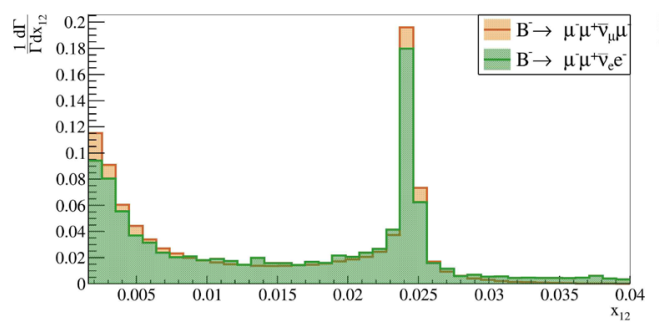

a)

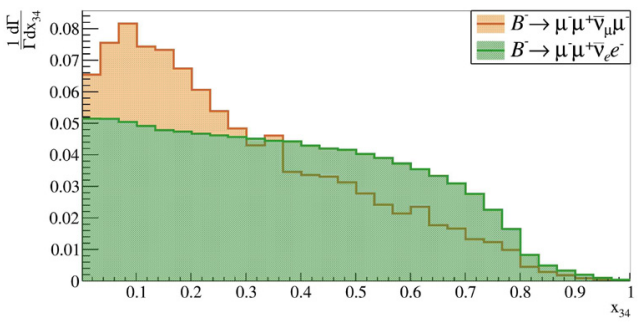

b)

Figure 4. Normalized differential distributions $\frac{1}{\Gamma} \frac{d \Gamma}{d x_{12}}$ a) and $\frac{1}{\Gamma} \frac{d \Gamma}{d x_{34}} \mathbf{b}$ ) for decays $B^{-} \rightarrow \mu^{+} \mu^{-} \bar{v}_{e} e^{-}$and $B^{-} \rightarrow \mu^{+} \bar{v}_{\mu} \mu^{-} \mu^{-}$.

Here we can obtain a photon pole for $x_{12} \rightarrow x_{12 \min }=\left(2 m_{\mu} / M_{1}\right)^{2}=0.0016$ and a peak from the $\omega(782)$ resonance for $x_{12} \rightarrow\left(M_{\omega} / M_{1}\right)^{2} \approx 0.023$. Due to the fact that the $\rho^{0}(770)$ meson has a width of about $150 \mathrm{MeV}$, the contribution from this meson in Fig. 4 (a) appears as a wide background to the narrow peak of the $\omega(782)$ resonance. The distributions by $x_{12}$ are given in the range [0, 0.04], which corresponds to the area of applicability of the VMD model for the considered decays in accounting $\rho^{0}$ and $\omega$ resonances alone. 


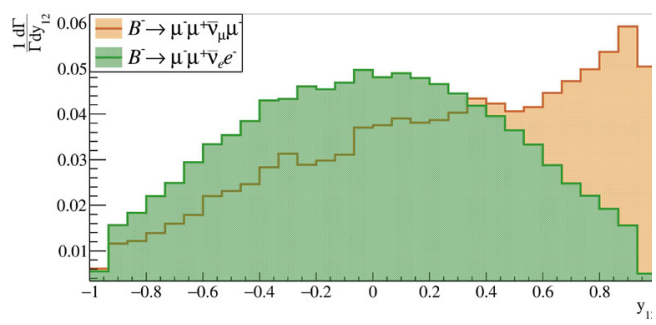

a)

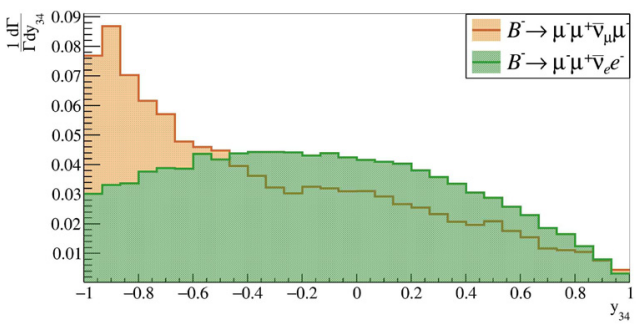

b)

Figure 5. Normalized differential distributions $\frac{1}{\Gamma} \frac{d \Gamma}{d y_{12}}$ a) and $\left.\frac{1}{\Gamma} \frac{d \Gamma}{d y_{34}} \mathbf{b}\right)$ for the decays $B^{-} \rightarrow \mu^{+} \mu^{-} \bar{v}_{e} e^{-}$ and $B^{-} \rightarrow \mu^{+} \bar{v}_{\mu} \mu^{-} \mu^{-}$.

From the distributions by $x_{34}$ in Fig. 4 (b) one infers an interesting feature: distributions for the identical and non identical particles in final state have different shape, highlighting the importance of the accounting Fermi antisymmetry in the decay $B^{-} \rightarrow \mu^{+} \bar{v}_{\mu} \mu^{-} \mu^{-}$. There are no poles in this channel, in accordance with the matrix element structure.

The difference of the same nature may be seen in the angular distributions by $y_{12}=\cos \theta_{12}$ and $y_{34}=\cos \theta_{34}$, which are presented in Fig. 5. Here the $\theta_{12}$ is the angle between the momentum of the positively charged lepton and the direction of the $B$-meson in the rest frame of the $\ell^{+} \ell^{-}-$pair and $\theta_{34}$ is the angle between the direction of the antineutrino and the direction of the $B$-meson in the rest frame of $\ell^{\prime} \bar{v}_{\ell^{\prime}}$-pair.

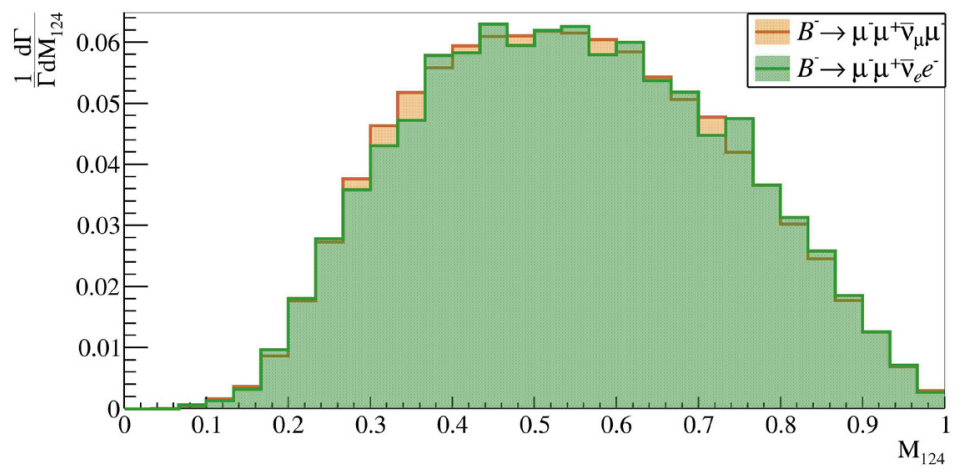

Figure 6. Normalized differential distributions $\frac{1}{\Gamma} \frac{d \Gamma}{d x_{124}}$ by invariant mass of all of the charged leptons in the final state for the decays $B^{-} \rightarrow \mu^{+} \mu^{-} \bar{v}_{e} e^{-}$and $B^{-} \rightarrow \mu^{+} \bar{v}_{\mu} \mu^{-} \mu^{-}$.

Detectability of the multi-lepton decays of the $B$-mesons with a neutrino in the final state may be linked to the distributions by normalized invariant mass of the charged leptons. The square of the corresponding mass is defined as:

$$
x_{124}=\frac{\left(k_{1}+k_{2}+k_{4}\right)^{2}}{M_{1}^{2}} \text {, }
$$


where the $k_{i}$ are four-momenta of charged leptons in the final state. The distributions by $x_{124}$ are presented in Fig. 6. One can see that the shape of the distribution by $x_{12}$ is not very sensitive to the existence of identical particles in the final state.

\section{Conclusions}

- We obtained the theoretical predictions for the branching ratios of the decays $B^{-} \rightarrow \mu^{+} \mu^{-} \bar{v}_{e} e^{-}$and $B^{-} \rightarrow \mu^{+} \bar{v}_{\mu} \mu^{-} \mu^{-}$in the framework of Standard Model and considered the effect on the results of the non - perturbative relative phase between the resonances;

- We examined the effects of the Fermi antisymmetry on the amplitude of the four - leptonic B - meson decays;

- We presented a set of experimentally useful differential distributions for the decays $B^{-} \rightarrow \mu^{+} \mu^{-} \bar{v}_{e} e^{-}$and $B^{-} \rightarrow \mu^{+} \bar{v}_{\mu} \mu^{-} \mu^{-}$in the framework of the Standard Model and discussed their characteristics.

\section{Acknowledgements}

The work was supported by grant 16-12-10280 of the Russian Science Foundation. The authors (A.Danilina and N.Nikitin) express their gratitude for this support.

\section{References}

[1] R. Aaij et al. [LHCb Collaboration], "Search for the rare decay $B^{+} \rightarrow \mu^{+} \mu^{-} \mu^{+} v_{\mu}$ ", Eur. Phys. J. C 79, no. 8, 675 (2019).

[2] A.V. Danilina and N.V.Nikitin, "Four-Leptonic Decays of Charged and Neutral $B$ Mesons within the Standard Model”, Phys. Atom. Nucl. 81, no. 3, 347 (2018) [Yad. Fiz. 81, no. 3, 331 (2018)].

[3] D. Melikhov and B. Stech, "Weak form-factors for heavy meson decays: An Update", Phys. Rev. D 62, 014006 (2000). 